\title{
A SINGLE-MEMBRANE PERMEATION-BASED SENSOR CONCEPT FOR THE MEASUREMENT OF MULTIPLE GASES
}

\author{
S. Schwebke ${ }^{1,2^{*}}$, V. Bazargan ${ }^{l}$, and B. Stoeber ${ }^{l}$
}

${ }^{1}$ The University of British Columbia, Vancouver, B.C., Canada

${ }^{2}$ Ilmenau University of Technology, Ilmenau, Germany

\begin{abstract}
A new concept for a gas sensor for measuring the partial pressures of multiple gases is demonstrated. The system is based on the pressure changes in two chambers, caused by the selective permeation of gases through a connecting membrane. A mathematical model based on permeation flow is developed to formulate measurement equations. Based on this model, a system for the measurement of specific gases that are typically dissolved in water is designed. A microfluidic multilayer polydimethylsiloxane (PDMS) device is fabricated for a proof of concept study. In experiments with air and nitrogen gas mixtures, pressures are recorded as a function of time, and this data is analyzed to calibrate the system and to determine the concentration of gases in a mixture.
\end{abstract}

\section{INTRODUCTION}

One important parameter in the assessment of water quality is the total amount of dissolved gas in water. Gas extraction probes that use a membrane to extract gases from water and measure their total pressure are already commercially available, and they are commonly used to assess the water quality of lakes and ponds. For more advanced water quality management there is a need for a device that can measure the concentration of individual gases dissolved in water, in particular oxygen, nitrogen, and carbon dioxide, which typically occur at the highest partial pressures.

Lazik and Geistlinger [1] proposed a permeation-based method with $m$ membranes to measure the partial pressures of $n$ $(\leq m)$ different gases by observing approximately linear pressure changes across these membranes and solving a system of linear equations. In contrast, our method allows for the measurement of the partial pressures of several gases in a mixture using a single membrane and at least one pressure sensor. It uses the solution of a system of permeation equations or parameter fitting to find the initial gas fractions. Both methods rely on a difference in membrane permeability for different gases.

We establish a mathematical model for our system based on the underlying physics, and we use this model to design a proof of concept device. The device is fabricated with processes commonly used in microfluidics. Permeation experiments with different mixtures of air and nitrogen are conducted to validate the model and demonstrate the measurement principle.

\section{SENSOR FUNCTION AND MODEL}

\section{Sensor Structure and Measurement Procedure}

Figure 1 shows our sensor concept, which includes two chambers of volumes $V_{s}$ and $V_{r}$ separated by a membrane of thickness $d$, area $A$ and permeability $P$, one pressure sensor for each chamber measuring $p_{s}(t)$ and $p_{r}(t)$ and inlets and outlets that can be opened and closed by valves.

\begin{tabular}{|c|c|c|}
\hline sample chamber & & reference chamber \\
\hline$p_{S}(t) \quad A$ & $d$ & $p_{r}(t)$ \\
\hline$J(t)$ & & $n_{r, 0}$ \\
\hline$V_{S}$ & $\mathcal{P}$ & $V_{r}$ \\
\hline
\end{tabular}

Figure 1: Two-chamber system with membrane.

The measurement procedure follows the sequence:

1. The reference chamber $\left(V_{r}\right)$ is flushed with a reference gas or a gas mixture of known composition (e. g. air), and it is then closed off.

2. The sample chamber $\left(V_{s}\right)$ is flushed with the gas mixture of interest and closed off.

3. The chamber pressures are measured over a certain amount of time (depending on time constants of the permeation processes).

4. Our algorithm is applied to the pressure signals to determine the initial gas volume fractions in the inflow chamber.

5. The system can be re-initialized by purging both chambers.

\section{Mathematical Model}

The mathematical model of the permeation process is based on a single-gas molar permeation flux driven by the pressure difference, proportional to permeability $P$ and membrane surface $A$ and inversely proportional to membrane thickness $d$ :

$$
J=P A \frac{p_{s}(t)-p_{r}(t)}{d}
$$

For a system of given initial conditions as shown in Figure 1, we can use (1) and the law of ideal gases, $p V=n R T$, to describe the reference chamber pressure

$$
p_{r}(t)=\frac{R T}{V_{r}}\left(n_{r, 0}+\int_{t_{0}}^{t} J(\tau) \mathrm{d} \tau\right)
$$

Here, the molar amount $n_{r}(t)$ is made up by the initial molar amount $n_{r, 0}$ plus the permeation flux over time into the chamber. Differentiating (2) with respect to time yields a differential equation governing the pressure changes over time; its solution is

$$
p_{r}(t)=\left(p_{r, 0}-p_{r, e}\right) \mathrm{e}^{-k t}+p_{r, e}
$$

with

$$
k=R \operatorname{TPA} \frac{V_{r}+V_{s}}{V_{r} V_{s} d}, \quad p_{r, e}=\frac{p_{r, 0} V_{r}+p_{s, 0} V_{s}}{V_{r}+V_{s}},
$$

where $p_{r, 0}$ and $p_{s, 0}$ are the initial pressures for the chambers and $p_{r, e}$ is the final equilibrium pressure (identical for both chambers).

This model is extended to multiple-gas systems using Dalton's law of partial pressures, 


$$
p=\sum_{k=1}^{n} p_{k}
$$

which is valid for gases of limited solubility in the membrane (Lacey and Loeb [2]). This allows us to describe the total reference chamber pressure $p_{r}(t)$ as the sum of the individual pressures $p_{r, a}(t), p_{r, b}(t)$ etc.; the individual partial pressures change in a different way due to the different gas permeabilities $P_{k}$ of the membrane and the different initial partial pressures in the two chambers.

Figure 2 shows predicted pressure signals $p_{r}(t)$ over time for a system of three gases with different initial gas fractions, based on our model.

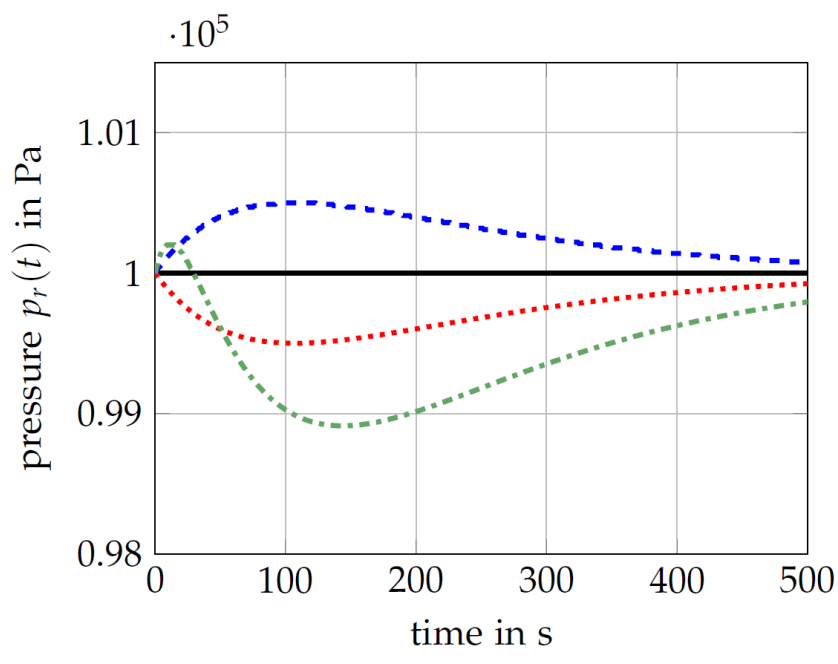

Figure 2: Reference chamber pressure over time for different fractions of up to three sample gases in the sample chamber. Reference chamber: $78 \% \mathrm{~N}_{2}, 22 \% \mathrm{O}_{2}$. Black, solid: equal gas mixture and initial pressure in both chambers; blue, dashed: $74 \%$ $\mathrm{N}_{2}, 26 \% \mathrm{O}_{2}$; red, dotted: $82 \% \mathrm{~N}_{2}, 18 \% \mathrm{O}_{2}$; green, dash-dotted: $89 \% \mathrm{~N}_{2}, 8 \% \mathrm{O}_{2}, 3 \% \mathrm{CO}_{2}$.

From (3) we can see that in general, a permeation function for a single gas $a$ can be written in the form

$$
p_{r, a}=p_{r, a, 0} \cdot a_{1}(t)+p_{s, a, 0} \cdot a_{2}(t),
$$

where

$$
a_{1}(t)=\mathrm{e}^{-k_{a} t} \cdot\left(1-\frac{V_{r}}{V_{r}+V_{s}}\right)+\frac{V_{r}}{V_{r}+V_{s}}, \quad a_{2}(t)=\frac{V_{s}}{V_{r}+V_{s}}\left(1-\mathrm{e}^{-k_{a} t}\right) .
$$

In our measurement process, the sum of the partial pressures of $n$ gases form the total pressure $p_{r}$, and this problem has $n$ unknown variables $\left(p_{s, a, 0}, p_{s, b, 0}\right.$, etc. $)$. If we assume $n$ pressure measurements $p_{r}\left(t_{i}\right)$ at time points $t_{i}$, we can establish a system of $n$ equations to solve for these unknowns. If we write (5) in the form

$$
p_{r, a}-p_{r, a, 0} \cdot a_{1}(t)=a_{2}(t) \cdot p_{s, a, 0},
$$

the system of equations for $n$ gases and $n$ time points can be written in the corresponding matrix form:

$$
p_{r}-A_{1} \cdot p_{r, 0}=A_{2} \cdot p_{s, 0},
$$

where $p_{r}$ is the vector of the total pressure measurements $p_{r}\left(t_{i}\right)$ for the reference chamber, $\boldsymbol{p}_{\boldsymbol{r}, \boldsymbol{\theta}}$ is the vector of the known initial reference chamber partial pressures, $\boldsymbol{A}_{1}$ and $\boldsymbol{A}_{2}$ are permeation matrices of the time dependent coefficients $a_{1}\left(t_{i}\right)$ and $a_{2}\left(t_{i}\right)$, and $\boldsymbol{p}_{\boldsymbol{s}, \boldsymbol{\theta}}$ is the vector of the initial sample chamber partial pressures, which are the desired measurement result. If all permeabilities $P_{k}$ for the different gases are different from one another, $\boldsymbol{A}_{2}$ can be inverted in order to yield the measurement equation

$$
p_{s, 0}=A_{2}^{-1} \cdot\left(p_{r}-A_{1} \cdot p_{r, 0}\right) \text {. }
$$

That means that, as expected, gases of identical permeability cannot be discerned. For practical purposes, the sensor concept can be used to measure the sum of the partial pressures of such gases.

In an experimental investigation, either the measurement equation (7) or a curve fit to our permeation model can be used to evaluate the data and find all inflow gas fractions.

\section{DESIGN, FABRICATION AND EXPERIMENTS Design of a Proof of Concept Device}

A material that offers sufficient selectivity (higher permeability of one gas over another) between nitrogen, oxygen and carbon dioxide is polydimethylsiloxane (PDMS). Compared to other polymers it also has a high gas permeability (Merkel et al. [3]), which makes it a desirable membrane material for our concept.

PDMS is often used for the fabrication of microfluidic devices, and thin layers can be achieved with commonly used fabrication processes.

A membrane thickness of $d=40 \mu \mathrm{m}$ is chosen in order to achieve permeation time constants in the range of a few minutes. Since the expected pressure differences during the measurement process can reach up to $2000 \mathrm{~Pa}$ as shown in Figure 2, the thin membrane has to be supported. For this purpose, a narrow grid of rectangular support pillars on either side of the membrane is designed for this two-layer PDMS device.

To open and close the inlet and outlet channels, microfluidic valves are included, as described by Unger et al. [4]. These valves are made from crossing channels in the two device layers - when a pressure is applied to one of the channels (control channel), the thin membrane separating the channels deflects and blocks the other channel.

Assuming that the pressure measurements as well as the membrane properties possess an uncertainty, the combined uncertainty for the measured gas fractions was calculated from Equation (7). This study was used to optimize the sensor geometry for a minimum measurement error.

The parameters of the final system are shown in Table 1. The expected time constants are based on typical permeability values from literature [3].

Table 1: System design parameters

\begin{tabular}{lllll}
\hline$d(\mu \mathrm{m})$ & $A\left(\mathrm{~cm}^{2}\right)$ & $V_{s}=V_{r}\left(\mathrm{~cm}^{3}\right)$ & $\tau_{N_{2}}(\mathrm{~min})$ & $\tau_{O_{2}}(\mathrm{~min})$ \\
\hline 40 & 0.2 & 0.125 & 6.5 & 3.2 \\
\hline
\end{tabular}

\section{Device Fabrication}

The two layers that make up the system are shown in Figure 3; the lower layer includes the membrane and its bottom support pillars (not shown). The mask design in Figure 4 shows the inlet and an outlet of the sample gas chamber that allows the sample gas to be flushed in quickly.

Attached to both chambers are wide sensor connector areas to 
allow connecting the external pressure sensors.

The fabrication steps follow the standard procedure for fabricating multi-layer soft lithography devices described in [5] and consist of spin-coating a $10 \mu \mathrm{m}$ thick photoresist layer on one silicon wafer for each of the two layers, structuring them via lithography, spin-coating the bottom layer with $50 \mu \mathrm{m}$ PDMS and pouring a thick PDMS layer (ca. $1 \mathrm{~cm}$ ) on the other wafer. The layers are cured at a temperature of $80^{\circ} \mathrm{C}$ for 45 minutes, and then the thick top layer is cut into individual chips which are bonded to the bottom layer after surface activation in air plasma. The bonded system is cured again, and then it is cut and peeled off the silicon wafer. All holes for in-/outlets are punched using a biopsy punch and the systems are finally bonded to glass substrates after a final air-plasma activation.

A finished device can be seen in Figure 5. Pressure sensors and gas supply for inlets and control channels are externally connected via Tygon tubing.

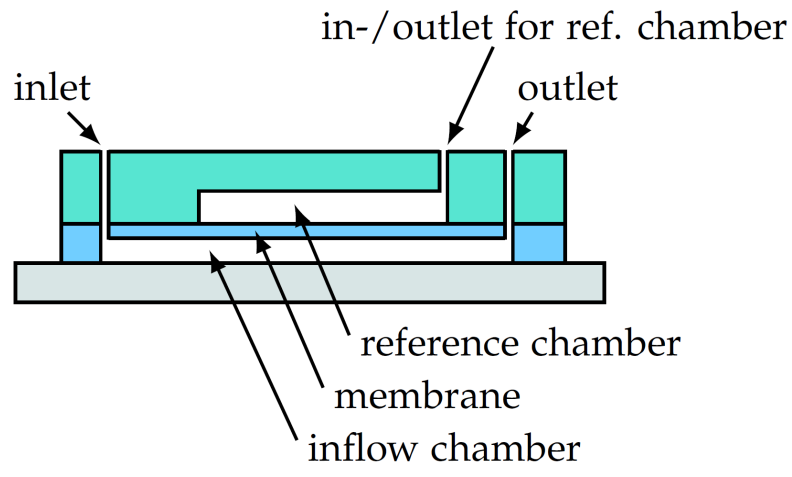

Figure 3: Schematic of the microfluidic PDMS device layers.

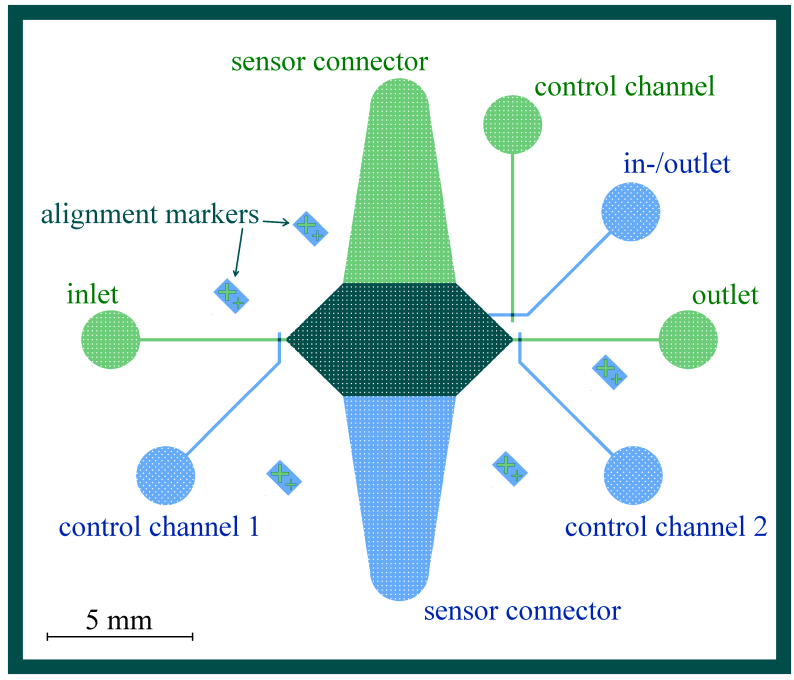

Figure 4: Mask design for the two-layer device. Colored segments are open channels/volumes within the system. The central hexagon is the $40 \mu \mathrm{m}$ thick membrane.

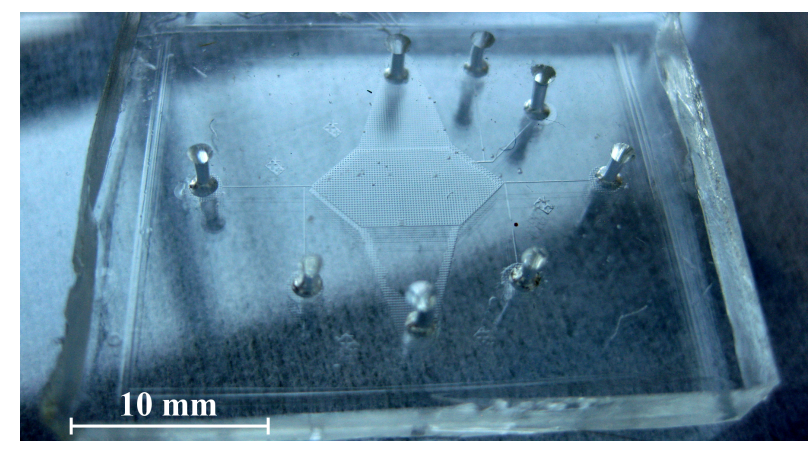

Figure 5: A device fabricated in multi-layer soft lithography using the masks in Figure 4.

\section{Permeation Experiments}

Each chamber was connected to a differential pressure sensor; in addition, the absolute pressure was also monitored during an experiment. The sample gas was connected to the inlet and controlled by the internal microfluidic valves of the device, which were actuated by a multi-channel pressure control system with control pressures up to $10^{5} \mathrm{~Pa}$.

For the permeation measurements, the reference chamber was filled with air at ambient pressure as the reference gas. The sample chamber was filled with the sample gas mixture, and then closed off using the valves. The permeation process between the sample chamber at an initial pressure slightly above the ambient pressure and the reference chamber at ambient pressure was then observed via the absolute chamber pressures. Air (ca. $\left.78 \% \mathrm{~N}_{2}, 21 \% \mathrm{O}_{2}\right)$ and nitrogen were used as sample gases.

For the air permeation test, the reference chamber was filled with air at ambient pressure and was left open, whereas the sample chamber was filled with air at a pressure of about $1500 \mathrm{~Pa}$ above the ambient pressure. We used our two-gas permeation model:

$$
p_{s}(t)=\left(p_{s, O_{2}, 0}-p_{s, O_{2}, e}\right) \mathrm{e}^{-k_{O_{2}} t}+\left(p_{s, N_{2}, 0}-p_{s, N_{2}, e}\right) \mathrm{e}^{-k_{N_{2}} t}
$$

based on Equation (3) to fit the measured pressure over time using the oxygen permeability, the nitrogen permeability and the reference chamber volume as curve fit parameters. It was found that the permeation time constants were affected by the diffusion path between the internal sensor volume and the membrane; for this reason the connector tubing between the two was kept as short as possible (ca. $2 \mathrm{~cm}$ ).

The air permeation result is shown in Figure 6. The fit result gives a chamber volume of $V_{s}=0.17 \mathrm{~cm}^{3}$, which is dominated by the pressure sensor's internal volume $\left(0.11 \mathrm{~cm}^{3}\right.$ according to the manufacturer) and the volume of the tubing.

We found the following permeability estimates: $P_{N_{2}} \approx 730$ Barrer, $P_{O_{2}} \approx 1480$ Barrer $\left(1\right.$ Barrer $\left.=3.30 \times 10^{-16} \mathrm{~mol} \mathrm{~mm}^{-2} \mathrm{~s}^{-1} \mathrm{~Pa}^{-1}\right)$. The corresponding time constants are $\tau_{N 2}=7.7 \mathrm{~min}$ and $\tau_{O 2}=3.8$ min. The selectivity ratio $P_{\mathrm{O}_{2}} / P_{\mathrm{N}_{2}} \approx 2$ is similar to literature values [3], but the absolute permeabilities are larger than most values from literature. The membrane thickness was not measured separately, so it is likely that the permeabilities just appear larger due to the membrane being thinner than assumed for the calculation. In effect this can be neglected since the ratio $P_{k} / d$ governs the exponential permeation term. 


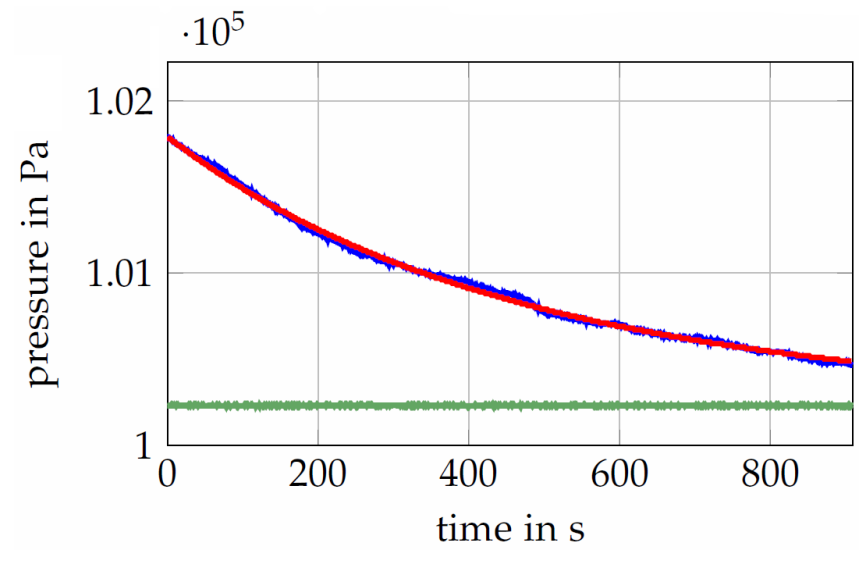

Figure 6: Results from the air permeation test with the microfluidic device. Green: pressure in the reference chamber. Blue: pressure in the sample chamber. Red: curve fit to the analytical model.

In another experiment, nitrogen was added into the inflow chamber. We used the previously identified permeabilities and chamber volumes to fit the measured pressure over time to our model with the initial gas fractions in the sample chamber as parameters. As expected, an elevated nitrogen level was identified. A good fit was achieved with $81.8 \% \mathrm{~N}_{2}$ and $18.2 \% \mathrm{O}_{2}$. A plot of the result is shown in Figure 7.

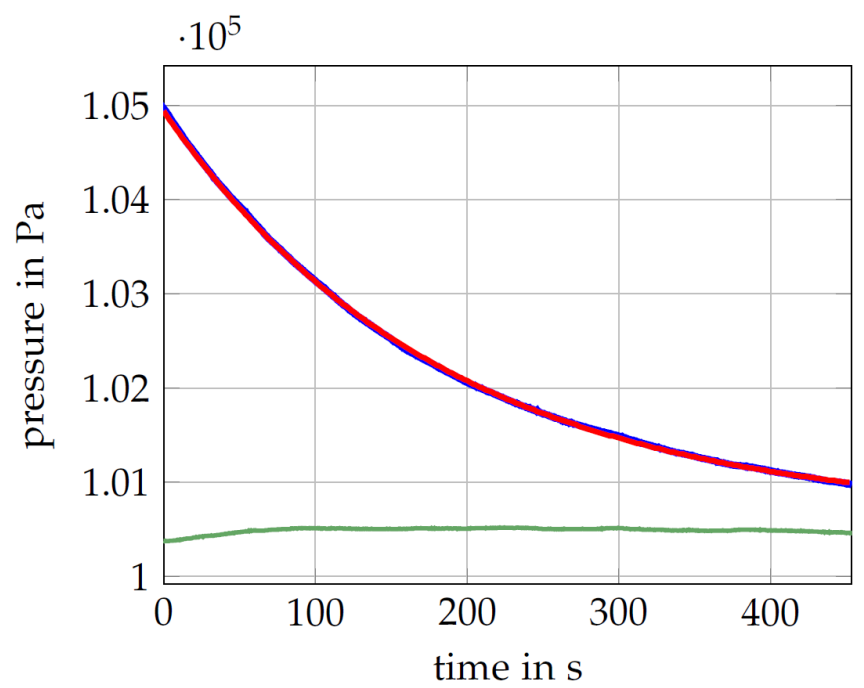

Figure 7: Nitrogen permeation test. Green: pressure in the reference chamber. Blue: pressure in the sample chamber. Red: a curve fit of the sample chamber pressure to the analytical model identifies the initial conditions: $81.8 \% \mathrm{~N}_{2}, 18.2 \% \mathrm{O}_{2}$. Note: The inflow chamber was initially pressurized with respect to the reference chamber, unlike in the simulation in Fig. 2 with identical initial pressures.

\section{CONCLUSIONS}

Based on literature and existing models of the permeation of gases through thin membranes, we derived a model for the permeation of multiple gases. The inversion of the permeation matrix leads to measurement equations that show the viability of a single-membrane sensor for multiple gases.

A microfluidic device with a PDMS membrane between two chambers was fabricated. The pressure changes during the permeation of different two-gas mixtures were measured.

The measurement data was used to find curve fits; a good agreement between model and experiment was found. After using one permeation experiment to identify the permeabilities of the membrane for oxygen and nitrogen and the chamber volume, these parameters were used in a permeation process with unknown initial partial pressures to find an estimate for the unknown gas fractions.

It was found that the connecting tubing to the external pressure sensors dominated the system volume and therefore the time constants of the permeation process between the chambers. This delay could be reduced greatly by bringing the sensors closer to the chambers, e.g. by integrating them on the substrate in future designs.

While a PDMS membrane offers the potential to measure partial pressures of nitrogen and oxygen (as well as carbon dioxide fractions in the order of magnitude of at least $1 \%$ ), materials with different gas selectivities could be chosen for a sensor system for the measurement of other gases.

\section{REFERENCES}

[1] D. Lazik, H. Geistlinger, "A new method for membrane-based gas measurements," Sensors and Actuators A: Physical, 117, pp. 241-251 (2005).

[2] R. E. Lacey, S. Loeb, "Industrial Processing with Membranes," John Wiley \& Sons, p. 299 (1972).

[3] T. C. Merkel et al., "Gas Sorption, Diffusion, and Permeation in Poly(dimethylsiloxane)," Journal of Polymer Science Part B: Polymer Physics, 38, no. 3, pp. 415-434 (2000).

[4] M. A. Unger et al., "Monolithic Microfabricated Valves and Pumps by Multilayer Soft Lithography," Science, 288, no. 5463, pp. 113-116 (2000).

[5] V. Bazargan, "Micro Flow Control Using Thermally Responsive Polymer Solutions," Master Thesis, University of British Columbia, October 2008.

\section{CONTACT}

*s.schwebke@gmail.com 